\title{
El agua en zonas rurales de México. Desafíos de la Agenda 2030
}

\author{
The Water in Mexico Rural Zones. \\ Challenges of The 2030 Agenda
}

\author{
Denise Soares* \\ doi: https://doi.org/10.31644/ED.V8.N2.2021.A09
}

\begin{abstract}
Resumen: El limitado acceso a los servicios de agua en las zonas rurales de México pone en riesgo el cumplimiento de la Agenda 2030 en su Objetivo de Desarrollo Sostenible número seis, relativo al agua y su saneamiento. El presente estudio, realizado en dos localidades rurales del estado de Puebla, tiene como fin identificar el acceso al agua por las familias y las estrategias empleadas para tratar de resolver la carencia de disponibilidad del recurso hídrico. Entre los resultados, se identificaron diferentes formas de abastecimiento y que la responsabilidad de disponer de agua recae en las mujeres. Se concluye argumentando que se requieren cambios estructurales profundos en el sector hídrico gubernamental, poniendo el enfoque de los derechos humanos como eje de la lógica de gestión.
\end{abstract}

Palabras clave: derecho humano al agua, desigualdades territoriales, objetivos de desarrollo sostenible (ODS), comunidades rurales, exclusión social.

Abstract: The limited access to water services in rural areas of Mexico puts at risk the fulfillment of 2030 Agenda in Sustainable Development Goal Number Six, related to water and sanitation. The present study, carried out in two rural localities in the state of Puebla, aims to identify access to water by families and the strategies used to try to solve the lack of availability of water resources. Among the results, different forms of supply were identified and that the responsibility for having water rests with women. It concludes by arguing that profound structural changes are required in the government water sector, putting the human rights approach as the axis of water management logic.

Keywords: human right to water, territorial inequalities, sustainable development goals (SDG), rural communities, social exclusion.

\footnotetext{
* Dra. en Antropología, investigadora del Instituto Mexicano de Tecnología del Agua (IMTA), área de participación social, México. ORCiD: http://orcid.org/0000-0003-1811-0139. Correo-e: denisefsoares@yahoo.com.mx.

Fecha de recepción: 01/09/2020. Fecha de aceptación: 10/06/2021. Fecha de publicación: 30/07/2021.

\section{(cc) BY-NC-ND}

EntreDiversidades. Revista de Ciencias Sociales y Humanidades, Vol. 8, Núm. 2 (17), julio-diciembre 2021. Páginas: 191-211 ISSN-e: 2007-7610. https://doi.org/10.31644/ED.V8.N2.2021.A09 


\section{Introducción}

México es un país con desigualdades en lo que se refiere a la disponibilidad natural de los recursos hídricos, debido a la dispersión de numerosas localidades rurales, las condiciones geográficas y topografía en donde se ubican múltiples asentamientos humanos. Mientras la región sur de nuestra nación se localiza en la zona intertropical del planeta, el norte se encuentra en la templada. El sureste, correspondiente a la tercera parte del país, es húmedo, con precipitaciones anuales que superan los $2000 \mathrm{~mm}$ por año; sin embargo, las otras dos terceras partes del territorio están catalogadas como áridas o semiáridas, con precipitaciones anuales menores a los $500 \mathrm{~mm}$. En total, México cuenta con el $0.1 \%$ del agua dulce del planeta, cifra bastante baja si consideramos que Brasil alcanza el 13\% (Nogueira, Milhorance y Mendes, 2020; SEMARNAT y CONAGUA, 2018).

Aunado a la escasa precipitación en una porción considerable del territorio mexicano, se está viviendo una sequía intensa, con consecuencias no solo para el acceso humano al agua, sino a la producción agropecuaria. En el año 2020 las lluvias no fueron suficientes para llenar las presas, y de las 210 con mayor volumen de almacenado más de la mitad se encuentra por debajo del $50 \%$ de su capacidad y el $30 \%$ posee menos del $25 \%$ de agua, en particular en el norte y centro del país. Junto a la sequía, que puede agravarse por los efectos del cambio climático, están los procesos sistemáticos de deforestación para cambio de uso de suelo, transformando bosques en campos o superficies urbanizadas, con consecuencias en la reducción de la capacidad de retención y de la disponibilidad de agua (Varela, Guerrero y Miguel, 2021).

El acceso a los servicios de agua no necesariamente se explica a partir de su disponibilidad natural, de tal suerte que nos encontramos frente a la paradoja de que justamente en el sur y sureste, en donde se encuentra el 69\% de los recursos hídricos del país, se cuenta con menor disponibilidad a dichos servicios en comparación con el norte, centro y noroeste, que disponen solo del 31\%. La paradoja no se da solamente en el ámbito del acceso a los servicios de agua, sino de distribución de la población y de la generación del Producto Interior Bruto (PIB). De hecho, las regiones con mayor disponibilidad de agua en el país cuentan con menor población ( $23 \%$ de la población total) y se genera el $13 \%$ del PIB, en comparación con las que presentan escasez de agua, en donde se concentra el $77 \%$ de la población y se produce el $87 \%$ PIB mexicano (SEMARNAT y CONAGUA, 2018; López, 2017; Vázquez-García, Pérez-Olvera y MuñozRodríguez, 2014).

Asimismo, esta desigualdad con relación al acceso a los servicios de agua no se da solamente en términos geográficos (diferenciación entre zonas norte, centro y sur del país), sino territoriales, ${ }^{1}$ vinculados con el nivel de urbanización de la región, de tal suerte que se presentan desigualdades

\footnotetext{
${ }^{1}$ Es relevante acercarnos a un planteamiento sobre los conceptos de región y territorio, toda vez que, teniendo sus orígenes en la geografía, ambos fueron apropiados por otros campos disciplinarios, en su búsqueda de brindar una dimensión espacial a las dinámicas sociales, en donde prevalecían enfoques temporales. El territorio es un referente empírico que ubica espacialmente las relaciones sociales que establecen los seres humanos en los ámbitos cultural, social, político o económico, por lo cual la acción social lo transforma continuamente. La región, a la par que el territorio, relaciona el entorno natural con la acción social de los seres humanos, por lo tanto, su diferenciación no se acota a determinismos geográficos. En el siglo XX el concepto de región se convirtió en la guía para el desarrollo de políticas públicas, a través de la clasificación de espacios y la priorización de regiones a partir de análisis de homogeneidades y diferencias (Llanos-Hernández, 2010).
}

EntreDiversidades. Revista de Ciencias Sociales y Humanidades, Vol. 8, Núm. 2 (17), julio-diciembre 2021. Páginas: 191-211 ISSN-e: 2007-7610. https://doi.org/10.31644/ED.V8.N2.2021.A09 
entre contextos rurales y urbanos, en donde la población rural se encuentra en situación evidente de desventaja. Al 2015, la cobertura de servicio de agua entubada en la vivienda o predio de zonas rurales era $12.2 \%$ inferior con relación a las urbanas (97.2\% urbanas y $85.0 \%$ rurales) (SEMARNAT y CONAGUA, 2018). ${ }^{2}$ Esta situación no es exclusiva de México, ya que tres de cada cuatro personas pobres en los países en desarrollo viven en las zonas rurales. Es evidente, entonces, que vivir en el sur de México y, además, en comunidades rurales, limita la posibilidad de acceder al agua. Entre los argumentos que suelen justificar dicha desigualdad de acceso a los servicios está lo relacionado con la sub-representación de lo rural frente a lo urbano, dado que más del 70\% de la población mexicana vive en ciudades con más de 2500 habitantes, por lo que la inversión pública no privilegia lo rural (Fernández L., Fernández y Soloaga, 2019; García y Vázquez, 2017).

El hecho de que las viviendas no cuenten con servicio continuo de agua impacta de manera diferencial a hombres y mujeres, incrementando la carga de trabajo de estas, quienes son las principales encargadas del manejo del agua al interior del hogar, debido a la tradicional división sexual del trabajo que adjudica a las mujeres las actividades relacionadas con la reproducción de las unidades domésticas, en las cuales el acceso al agua es imprescindible. Ello refuerza las desigualdades de género en las zonas rurales, por sobrecargar a las mujeres de trabajo doméstico y quitarles tiempo que podrían emplear para acceder a otras oportunidades (Gutiérrez, et al., 2013).

Podemos argüir que la heterogeneidad en el acceso a los servicios de agua, tanto en términos de regiones (norte y sur) como de niveles de urbanización (territorios rurales y urbanos), no se circunscribe al discurso oficial reiteradamente anunciado que presenta argumentos relacionados con la baja disponibilidad del recurso, los costos elevados de su conducción en las tuberías, la carencia de recursos financieros para el mantenimiento de la infraestructura, la obsolescencia de las redes, la falta de pago del servicio, el desperdicio del agua por los usuarios, entre otros. Por el contrario, está directamente asociada con las relaciones de poder, que crean la distribución desigual y el acaparamiento del agua, promoviendo la escasez del servicio concentrada en determinadas regiones, lo que genera segregación y desigualdad territorializada. Es decir, el agua no se gestiona como un bien público homogéneo, sino que existen profundas diferencias en su suministro, las cuales no están relacionadas con cuestiones técnicas sino con intereses empresariales y de actores privilegiados, quienes definen las zonas de buena y mala calidad del servicio, convirtiendo al agua entubada en un ente segmentador y excluyente del territorio (Vargas, Peña y Soares, 2018; Peña y Pérez, 2016). Entonces, el gran problema no es la disponibilidad del recurso, sino la desigualdad, que excluye a determinados grupos sociales del acceso a los servicios y retroalimenta el círculo vicioso de la pobreza. De hecho, estos autores se refieren al ciclo del agua como:

\footnotetext{
${ }^{2}$ Las estadísticas de cobertura de servicio de agua no reflejan el acceso real al recurso por las viviendas, sino solamente los quilómetros de tuberías construidos, motivo por el cual es imprescindible cambiar el sistema de indicadores de acceso al agua, para que se mida la accesibilidad, disponibilidad y calidad.
}

EntreDiversidades. Revista de Ciencias Sociales y Humanidades, Vol. 8, Núm. 2 (17), julio-diciembre 2021. Páginas: 191-211 ISSN-e: 2007-7610. https://doi.org/10.31644/ED.V8.N2.2021.A09 
[...] un sofisticado proceso hidrosocial que ofrece una paradoja, el agua de mejor calidad escurre, fluye o brota hacia los centros de poder económico e influencia política. Se aleja con facilidad de las casas humildes y parece preferir las zonas industriales, los complejos habitacionales de altos ingresos y los agronegocios globales (Peña y Pérez, 2016: 21).

Este ciclo del agua planteado desde las relaciones de poder deja claro que la desigualdad en México es un fenómeno complejo y multifuncional, con profundas raíces históricas que determinan oportunidades y trayectorias diferenciadas entre distintos grupos sociales, contribuyendo a profundizar las distancias. El rezago en el acceso al agua no se manifiesta de manera aislada, sino que se presenta como el resultado de la articulación de una serie de desigualdades, entre ellas la territorial (diferenciación entre lo urbano y rural), la económica, la étnica y la de género, provocadas por diversas causas y que se explican desde ámbitos personales, relacionales y estructurales que determinan las posibilidades de las personas para acceder, controlar y mantener recursos a lo largo de su vida. Por tanto, la desigualdad en el acceso al agua debe ser vista desde esta perspectiva estructural y no aceptando justificaciones simplistas y tecnicistas, por lo cual las políticas hídricas deben articularse con las sociales si realmente estamos comprometidos en abatirla. Asimismo, es imprescindible un cambio de enfoque, tanto en las políticas como en la práctica, orientado hacia el abordaje de las causas de la exclusión y la desigualdad. De hecho, México se ha comprometido con ello en los Objetivos de Desarrollo Sostenible (ODS), cuyo lema central es "no dejar a nadie atrás" (WWAP, 2019; Jusidman, 2009).

Este artículo busca ahondar en la discusión sobre los desafíos para el cumplimiento del ODS 6 - garantizar la disponibilidad y la gestión sostenible del agua y el saneamiento para todos- en México, en su meta uno, concerniente al agua potable. Si bien los ODS se refieren a agua potabilizada, para el caso de México hablaríamos solamente de "agua entubada", dado que la potabilidad del agua está en tela de juicio. Para ello, se obtuvo información empírica en relación con cuatro variables: acceso al agua, medios de transporte utilizados para el abasto del recurso hídrico, quién realiza la actividad de acarreo del agua y disponibilidad del agua para uso personal y doméstico. Para tal fin se presenta el estudio de dos comunidades rurales ubicadas en la sierra nororiental del estado Puebla, México, pertenecientes al municipio de Zautla, El Mirador y Micuahutla. El trabajo comprende cuatro secciones: primera, un breve enfoque histórico acerca de lo que representa para el país el cumplimiento de una agenda tan ambiciosa como lo son los ODS, en especial el ODS 6, relacionado con el agua y el saneamiento; segunda, correspondiente al acercamiento a la metodología utilizada y a la zona de estudio; tercera, en la que se comparten los resultados encontrados relacionados con el acceso y disponibilidad de agua, desde la perspectiva de los actores sociales locales; $y$, finalmente, las conclusiones.

EntreDiversidades. Revista de Ciencias Sociales y Humanidades, Vol. 8, Núm. 2 (17), julio-diciembre 2021. Páginas: 191-211 ISSN-e: 2007-7610. https://doi.org/10.31644/ED.V8.N2.2021.A09 


\section{Los desafíos del ODS 6 en México}

No están siendo pocos los esfuerzos en aras de reducir las brechas de desigualdad no solo en México, sino a nivel mundial. De hecho, la Organización de las Naciones Unidas vienen liderando una serie de iniciativas, entre ellas los Objetivos de Desarrollo del Milenio (ODM), con propósitos de desarrollo humano fijados en el año 2000, cuyas metas debían cumplirse en el 2015. Los ODM se estructuraron en ocho objetivos y 21 metas, siendo que la meta diez del objetivo siete — garantizar la sostenibilidad del medio ambiente — se refería a reducir a la mitad, para el año 2015, el porcentaje de personas que carecían de acceso sostenible a agua potable y a saneamiento básico. En la evaluación de los logros de los ODM en materia de agua y saneamiento, de los 189 países miembros de Naciones Unidas que acordaron participar en dicho desafío, 151 cumplieron la meta de agua y 98 de saneamiento. México reportó el cumplimiento de ambas metas, incrementando en $12 \%$ el acceso al agua en el periodo de 1990 a 2015 (82\% a $94 \%$ ) y en $27 \%$ el acceso al saneamiento (66\% a 93\%) (Sandoval, 2017; SEMARNAT y CONAGUA, 2018).

Sin embargo, el Relator Especial de Naciones Unidas sobre los derechos al agua potable y al saneamiento, en su misión a México realizada en mayo de 2017, puso en entredicho los resultados que el país ha anunciado respecto a los ODM, argumentando que las cifras presentadas no reflejan el acceso efectivo a los servicios por parte de las poblaciones, sino solamente la existencia de alguna forma de infraestructura, aseverando que la instalación de líneas de conducción de agua y drenaje no garantiza el ejercicio de los derechos humanos en la materia, desde los referentes de suficiencia, continuidad, calidad, aceptabilidad y asequibilidad; de tal suerte que las limitaciones en la prestación de dichos servicios minan significativamente su contribución al bienestar de la población. Asimismo, reforzando los planteamientos del Relator Especial, el Programa de Monitoreo Conjunto (PMC) de la Organización Mundial de la Salud (OMS) y el Fondo de Naciones Unidas para la Infancia (UNICEF) ha asegurado que si México hubiera tomado en consideración las tres variables propuestas por los Objetivos de Desarrollo Sostenible 3 para la medición del indicador de acceso al agua, el resultado alcanzado en términos de cobertura del acceso efectivo a este recurso sería de tan solamente el 43\%, es decir, el país estaría reprobado en su meta de ODM (OHCHR, 2017; OMS y UNICEF, 2017).

Los ODS constituyen una herramienta que ayuda a fortalecer las acciones implementadas con anterioridad por los Estados miembros de las Naciones Unidas en el marco de los esfuerzos para la obtención de los ODM. Son 17 objetivos globales para erradicar la pobreza, proteger el planeta y asegurar la prosperidad de todas las personas, fijados en el año 2015 y con meta de cumplirse en el 2030. A los 17 objetivos se asocian 169 metas y 230 indicadores. El agua ha

\footnotetext{
${ }^{3}$ El ODS 6, referente a "Garantizar la disponibilidad y la gestión sostenible del agua y el saneamiento para todos", está conformado por seis metas, a saber: 1) agua potable, 2) saneamiento e higiene, 3) calidad del agua y aguas residuales, 4) uso de los recursos hídricos y escasez de agua, 5) gestión de los recursos hídricos, y 6) ecosistemas relacionados con el agua. Asimismo, hay dos metas consideradas aparte, enunciadas como "a” (cooperación internacional y creación de capacidades) y "b" (participación de las partes interesadas). La medición de la meta uno, relacionada con la cobertura del agua potable debe darse tomando en cuenta las tres variables del indicador 6.1: 1) el agua debe ser libre de contaminantes, 2) debe ser accesible en las instalaciones, y 3) estar disponible cuando sea necesaria (OMS y UNICEF, 2017).
}

EntreDiversidades. Revista de Ciencias Sociales y Humanidades, Vol. 8, Núm. 2 (17), julio-diciembre 2021. Páginas: 191-211 ISSN-e: 2007-7610. https://doi.org/10.31644/ED.V8.N2.2021.A09 
ganado un papel protagónico en esta nueva agenda de desarrollo, constituyéndose en el objetivo seis — garantizar la disponibilidad y la gestión sostenible del agua y el saneamiento para todos—, el cual cuenta a su vez con seis metas específicas y dos transversales. Los ODS proponen avances en términos de estrategias, convirtiéndolos en una agenda ambiciosa al presentar un enfoque orientado hacia los derechos humanos y al poner énfasis en la meta de universalidad del acceso a los servicios de agua y saneamiento y en la incidencia de manera concreta en la reducción de la desigualdad social. Vale recordar que el agua es imprescindible tanto para la realización de actividades productivas como reproductivas y, como tal, el cumplimiento del objetivo 6 de los ODS constituye un requisito de relevancia para el logro de los otros objetivos de la Agenda 2030 para el Desarrollo Sostenible, de tal suerte que el acceso al agua y al saneamiento es un fin en sí mismo y, a la par, un elemento que impulsa el desarrollo de otros ODS (Hortelano e Hidalgo, 2016; ONU-Agua, 2017).

México sostiene que viene generando las condiciones para cumplir con los ODS a través de la creación en el año 2015 del Comité Técnico Especializado de los Objetivos de Desarrollo Sostenible (CTEODS), a fin de coordinar la generación, seguimiento y actualización de los datos e indicadores de monitoreo de los avances en el cumplimiento de la Agenda 2030 a nivel nacional. De hecho, en su Informe Nacional Voluntario de 2018, denominado "Avance en el cumplimiento de la Agenda 2030", ha reportado una serie de avances ${ }^{4}$ y acciones emblemáticas 5 realizadas en el marco del cumplimiento del ODS 6, relativo al agua y al saneamiento. Sin embargo, han sido insuficientes las acciones y presupuestos para superar obstáculos estructurales para el logro de los ODS. Asimismo, los ODS tienen un enfoque de derechos y sería indispensable para la implementación de la Agenda 2030 la alineación del país a dicha perspectiva, dado que los derechos humanos al agua y al saneamiento ya están consagrados en la constitución mexicana (Sandoval y Seguin, 2018; Gobierno de la República, 2018).

Otro obstáculo para el cumplimiento de la Agenda 2030 en términos de agua y saneamiento es el hecho de que prevalece en el sector hídrico mexicano un modelo de gestión opaco, excluyente e insostenible, que no rinde cuentas a la ciudadanía, privilegia la mega infraestructura e intereses económicos sobre las necesidades de agua para uso personal y doméstico y permite la sobreexplotación y contaminación del recurso. Asimismo, el sector atraviesa por una severa crisis, en donde la gestión de los recursos hídricos padece de una serie de debilidades, entre ellas, la limitación presupuestal, la desarticulación institucional e interinstitucional, la corrupción, la

\footnotetext{
${ }^{4}$ Entre los avances relacionados con el acceso al agua, se reporta una elevada cobertura de agua entubada (94.5\%), así como de población que cuenta con acceso sostenible a una fuente de abastecimiento de agua (95.3\%). En términos de acceso al drenaje, según el informe, el 93.6\% de la población en viviendas particulares habitadas dispone de drenaje (Gobierno de la República, 2018).

${ }^{5}$ Las acciones emblemáticas presentadas en el marco del Informe Nacional Voluntario de 2018 en términos de avances en el cumplimiento de la Agenda 2030 se refieren a programas operados por Conagua, Comisión Nacional Forestal (Conafor) e Instituto Nacional de Pueblos Indígenas (INPI, anteriormente denominado Comisión Nacional para el Desarrollo de los Pueblos Indígenas-CDI) (Gobierno de la República, 2018). Dichos programas ya venían operando con anterioridad a los compromisos asumidos por el país con relación a los ODS y no tuvieron cambios significativos en aras de alinear sus indicadores a los propuestos por la plataforma de la Agenda 2030, por lo cual argüimos que México no se está esforzando lo suficiente para cumplir las metas del ODS 6.
}

EntreDiversidades. Revista de Ciencias Sociales y Humanidades, Vol. 8, Núm. 2 (17), julio-diciembre 2021. Páginas: 191-211 ISSN-e: 2007-7610. https://doi.org/10.31644/ED.V8.N2.2021.A09 
obsolescencia y el rezago en infraestructura, la carencia de diagnósticos actualizados y eficaces acerca de la situación real del acceso efectivo a los servicios de agua y saneamiento, la desigualdad de acceso a servicios entre zonas urbanas y rurales y la deficiencia en mecanismos y canales de participación ciudadana, entre otras carencias que contribuyen para la conformación de una muy débil estructura de gobernanza (Sandoval, 2017; Sandoval y Seguin, 2018).

Por ello, para avanzar en el logro del ODS 6 se requiere de cambios estructurales profundos, que van desde la lógica de la gestión, que deberá migrar hacia los derechos humanos al agua y al saneamiento, hasta una resignificación de las actuales estrategias de planeación, financiamiento, implementación y evaluación de las políticas, programas y acciones en torno al acceso de este recurso. Se necesitaría de la voluntad política para dar un giro sustancial al modelo de gestión hídrica, hacia la sostenibilidad ambiental, económica y social, con la promoción de la justicia hídrica. De hecho, el lema de la Agenda 2030 es "No dejar a nadie atrás", sin embargo, ya se quedaron atrás muchos grupos sociales en México, como indígenas, afrodescendientes, mujeres jefas de familia, habitantes de zonas periurbanas y rurales, entre otros colectivos en condiciones de vulnerabilidad, quienes sufren un mayor rezago en términos de acceso al agua y al saneamiento y que deberían tener la prioridad de atención en esta nueva agenda de desarrollo. En fin, se trata de desarrollar políticas hídricas democráticas y estrategias de desarrollo sostenibles orientadas a promover una distribución equitativa del agua en un marco de sostenibilidad ambiental, en coordinación con políticas sociales encaminadas a abatir el rezago social (Sandoval, 2017; Sandoval y Seguin, 2018).

Si bien los ODS marcan una ruta teóricamente enfocada en la reducción de las desigualdades, no se puede obviar que sus planteamientos no ponen en tela de juicio sus causas estructurales, toda vez que plantean una refuncionalización del modelo de crecimiento con medidas paliativas, lo que debilita la factibilidad de su implementación con éxito. Las alternativas de desarrollo presentes en una sociedad capitalista se orientan bajo el paradigma del mercado y, como tal, no es compatible con principios como justicia, derechos humanos, sostenibilidad e igualdad. Preceptos con los cuales los Sistemas de Naciones Unidas sistemáticamente tratan de maquillar los actuales modelos de desarrollo (Oliveira, et al., 2020).

La presente contribución está enfocada en el abordaje del cumplimiento de los desafíos del ODS 6, concerniente al agua potable, en dos comunidades rurales de México. Al describir las dificultades para acceder al agua en dichas localidades se rescatan evidencias empíricas acerca de los retos para el cumplimiento de la Agenda 2030 en nuestro país y enriquece el debate en la materia, al dar voz a los actores sociales en el territorio, permitiendo comprender sus relaciones con el agua y los desafíos para acceder al recurso. Ello puede constituirse en insumos para la territorialización de programas y proyectos orientados a ampliar la cobertura de los servicios de agua. Asimismo, se evidencia la necesidad de pensar el ODS 6 desde la perspectiva del ejercicio del derecho humano al agua.

EntreDiversidades. Revista de Ciencias Sociales y Humanidades, Vol. 8, Núm. 2 (17), julio-diciembre 2021. Páginas: 191-211 ISSN-e: 2007-7610. https://doi.org/10.31644/ED.V8.N2.2021.A09 


\section{Zona de estudio y estrategia metodológica}

Zautla se encuentra en la sierra nororiental de Puebla, que a su vez forma parte de la Sierra Madre Oriental, de tal suerte que el territorio del municipio presenta un relieve bastante montańoso e irregular, conformado por varias sierras y cerros aislados, así como por valles intermontanos, determinando continuos ascensos y descensos, con una altura sobre el nivel del mar que va desde mil 960 a los dos mil 700 metros. La temperatura oscila, durante el transcurso del año, entre $6^{\circ} \mathrm{C}$ y $24^{\circ} \mathrm{C}$, rara vez bajando a menos de $2^{\circ} \mathrm{C}$ o subiendo a más de $27^{\circ} \mathrm{C}$. El municipio es predominantemente rural, con un total de 49 localidades — solo una de ellas cuenta con más de 2500 habitantes, por lo que es considerada urbana en términos de población-. De hecho, la población es dispersa y 13 comunidades cuentan con menos de 100 habitantes, mientras 25 tienen de 100 a 499 personas. En términos de desigualdad, el municipio presenta un grado de rezago social y de marginación altos. ${ }^{6}$ Las dos comunidades de estudio, El Mirador y Micuahutla, cuentan con poca población, de hecho, mientras en El Mirador hay 117 personas ( 44 hombres y 73 mujeres), Micuahutla cuenta con 48 habitantes (23 hombres y 25 mujeres). Ambas localidades presentan alto grado de rezago social (INAFED, 2020; INEGI, 2016; SEDESOL, 2013). Para el desarrollo de este estudio se consultaron fuentes de información secundaria, con revisión bibliográfica sobre los temas de los Objetivos de Desarrollo Sostenible, desigualdades y su articulación con el desarrollo. Para la obtención de información empírica se utilizó una herramienta cuantitativa, consistente en el levantamiento de una encuesta con las siguientes variables: identificación de las personas informantes y acceso a los servicios de agua, tomando en cuenta la accesibilidad y disponibilidad.

La selección de ambas localidades para el desarrollo del estudio de caso se debe a los siguientes factores: la existencia de un sujeto social — formal o informal— con interés en los temas relacionados con el agua y el saneamiento; presencia de contactos que garantizan una relación de confianza para el desarrollo de la investigación inicial y la posibilidad de instrumentar un proyecto a más largo plazo (en la siguiente etapa de esta investigación se realizará la instalación de sistemas domiciliarios para el acceso al agua y al saneamiento en todas las viviendas, desde la perspectiva del ejercicio de los derechos humanos) y situaciones problemáticas relacionadas con el agua y al saneamiento. Se está trabajando de manera coordinada con la Asociación Civil de Desarrollo Centro de Estudios para el Desarrollo Rural (CESDER), la cual cuenta con alrededor de 30 años de arraigo e incidencia en la zona.

El trabajo de campo se realizó entre junio de 2019 y enero del 2020 y estuvo dividido en dos etapas. En la primera, se llevaron a cabo asambleas comunitarias en ambas localidades, convocadas

\footnotetext{
${ }^{6}$ La diferencia entre el Índice de Marginación y el Índice de Rezago Social está en el ámbito de medición, la institución que lo desarrolló y el número y calidad de sus variables. Mientras el Índice de Marginación posee una desagregación estatal y municipal, fue propuesto por el Instituto Nacional de Estadística y Geografía (INEGI) y considera los indicadores de educación, vivienda, ingreso por trabajo y la distribución de la población, el Índice de Rezago Social cuenta con desagregación que llega a nivel de localidad, fue desarrollado por el Consejo Nacional de Evaluación de la Política de Desarrollo Social (CONEVAL) y considera indicadores de educación, salud, servicios básicos, calidad y espacios de la vivienda y activos en el hogar (CONEVAL, 2020).
}

EntreDiversidades. Revista de Ciencias Sociales y Humanidades, Vol. 8, Núm. 2 (17), julio-diciembre 2021. Páginas: 191-211 ISSN-e: 2007-7610. https://doi.org/10.31644/ED.V8.N2.2021.A09 
por el Juez de Paz en El Mirador y líderes comunitarios en Micuahutla, ${ }^{7}$ a fin de solicitarles el permiso para trabajar con las mismas, presentando los objetivos y alcances de la investigación. Tras obtenerlo, se empezó a diseńar el instrumento para la recolección de la información. En la segunda etapa se procedió al levantamiento de una encuesta para conocer las estrategias locales de acceso a los servicios de agua en ambas localidades, tomando en cuenta las siguientes variables: fuentes de provisión del agua, medios para transportar el agua a las viviendas, número de viajes realizados para su abastecimiento, quiénes realizan la actividad de acarreo de agua, regularidad del servicio y disponibilidad del vital líquido. Se aplicaron encuestas a un representante por domicilio en cada localidad, cubriendo la totalidad de las viviendas habitadas (33 en El Mirador y nueve en Micuahutla), de tal manera que podemos afirmar que se ha realizado un censo en ambas localidades. De las 42 personas encuestadas, 20 son hombres y 22 mujeres.

\section{Acceso al agua en las dos localidades}

Mientras la localidad de El Mirador cuenta con un manantial ubicado alrededor de un kilómetro de distancia como fuente de agua —el cual abastece un sistema hidráulico que transporta el líquido a la comunidad y es almacenado en un tanque elevado, para su posterior distribución a las viviendas - Micuahutla no tiene ninguna fuente directa de abasto y depende de camiones pipa que el municipio envía regularmente. El agua es almacenada en una cisterna comunitaria a la que las familias deben acercarse para abastecerse, dado que no existe un sistema de distribución. El hecho de que no conozcan con seguridad el origen del agua que el municipio les brinda genera dudas sobre su calidad, motivo por el cual algunas familias compran garrafones de agua en la localidad. En contraposición, ningún habitante de El Mirador recurre a dicha práctica, por afirmar que su agua proviene de manantial y por ello es de buena calidad. Asimismo, la estrategia de contar con un sistema de captura de agua de lluvia, aunque instalado de manera muy artesanal, es más difundida y reconocida como necesaria en Micuhuatla que en El Mirador, quienes lo consideran como una alternativa que contribuye a reducir las carencias de la vivienda en términos de acceso al agua, por lo menos en las épocas de lluvia.

${ }^{7}$ Debido al reducido tamaño de la localidad de Micuahutla, esta no cuenta con Juez de Paz, que es la autoridad máxima a nivel comunitario, de tal suerte que son los propios líderes comunitarios quienes convocan a la asamblea y deciden de manera colectiva.

EntreDiversidades. Revista de Ciencias Sociales y Humanidades, Vol. 8, Núm. 2 (17), julio-diciembre 2021. Páginas: 191-211 ISSN-e: 2007-7610. https://doi.org/10.31644/ED.V8.N2.2021.A09 
Cuadro 1. Medios para proveerse de agua para uso doméstico en ambas localidades

\begin{tabular}{|l|l|r|r|}
\hline Localidad & \multicolumn{1}{|c|}{ Medios para provisión } & Frecuencia & Porcentaje \\
\hline \multirow{2}{*}{} & Red domiciliaria & 4 & 12.1 \\
\cline { 2 - 4 } & $\begin{array}{l}\text { Red domiciliaria, acarreo desde el manantial y } \\
\text { cosecha de agua de lluvia }\end{array}$ & 7 & 21.2 \\
\cline { 2 - 4 } & Red domiciliaria y acarreo desde el manantial & 9 & 27.3 \\
\cline { 2 - 4 } & Red domiciliaria y captura de agua de lluvia & 12 & 36.4 \\
\cline { 2 - 4 } & $\begin{array}{l}\text { Acarrea de la casa de su padre y captura de agua } \\
\text { de lluvia }\end{array}$ & 3.0 \\
\cline { 2 - 4 } & Total & $\mathbf{3 3}$ & $\mathbf{1 0 0 . 0}$ \\
\hline \multirow{2}{*}{$\begin{array}{l}\text { Cisterna colectiva y captura de agua de lluvia } \\
\text { domiciliaria }\end{array}$} & $\begin{array}{l}\text { Cisterna colectiva, captura de agua de lluvia } \\
\text { domiciliaria y compra de garrafón }\end{array}$ & 66.7 \\
\cline { 2 - 4 } & Total & 3 & 33.3 \\
\cline { 2 - 3 } & & $\mathbf{9}$ & $\mathbf{1 0 0 . 0}$ \\
\hline
\end{tabular}

Fuente: Elaboración propia.

Es importante resaltar que el sistema de El Mirador transporta el agua por medio de un desnivel de no menos de 700 metros, dado que el manantial está situado cuesta abajo, lo que no solo demanda un fuerte pago de energía eléctrica a la Comisión Federal de Electricidad (CFE), sino la necesidad de mantenimiento constante del sistema de succión de agua. Cuando la bomba de agua se estropea y no está en funcionamiento, alrededor del $50 \%$ de las personas encuestadas en El Mirador depende de idas al manantial para satisfacer su consumo habitual de agua. De hecho, en períodos de desperfecto de la bomba, que aunque no son frecuentes ocurren, la comunidad regresa a los remotos tiempos de acarreo del agua. El sistema de aguas abastece a todas las viviendas, con excepción de una, que está en lo más alto de la localidad, de tal suerte que esta familia acarrea el agua todo el tiempo, desde la casa del padre de la jefa del hogar, quien vive cerca.

Como se puede observar en el Cuadro 1, ante una red pública para el abasto de agua inexistente (Micuahutla) o deficiente (El Mirador), las familias se ven en la necesidad de acarrear el agua para contar con el vital líquido en sus viviendas. Hay diferencias significativas entre los medios de transporte utilizados en ambas localidades. Mientras El Mirador usa predominantemente transporte animal (burro y caballo) — de hecho, solo una familia asume que su único medio para el abasto de agua es la fuerza humana (a pie)—, en Micuahutla más de la mitad de las familias acarrea el agua caminando a sus hogares, valiéndose o no de carretillas. Estas diferencias entre los medios de transporte para acceder al agua se explican por la distancia de la comunidad a la fuente, dado que El Mirador está relativamente lejos del manantial, lo que dificultaría el transporte de agua cuesta arriba a pie, mientras que la cisterna colectiva de Micuahutla está adjunta a la localidad y solo las viviendas más alejadas tienen más dificultad para el acarreo sin el uso de transporte animal. Asimismo, las familias usan los medios mencionados, solos o en forma combinada, conforme puede observarse en el Cuadro 2.

EntreDiversidades. Revista de Ciencias Sociales y Humanidades, Vol. 8, Núm. 2 (17), julio-diciembre 2021. Páginas: 191-211 ISSN-e: 2007-7610. https://doi.org/10.31644/ED.V8.N2.2021.A09 


\section{Cuadro 2. Medios de transporte utilizados por las familias para abastecerse de agua en ambas localidades.}

\begin{tabular}{|c|c|c|c|}
\hline Localidad & $\begin{array}{c}\text { Medios de transporte para abastecerse } \\
\text { de agua }\end{array}$ & Frecuencia & Porcentaje \\
\hline \multirow{6}{*}{ 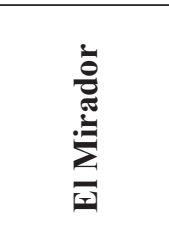 } & En burro o caballo & 20 & 60.6 \\
\hline & Caminando & 1 & 3.0 \\
\hline & En burro y caminando & 1 & 3.0 \\
\hline & Total & 22 & 66.7 \\
\hline & No opera & 11 & 33.3 \\
\hline & Total & 33 & 100.0 \\
\hline \multirow{6}{*}{ 冚 } & En burro o caballo & 2 & 22.2 \\
\hline & Caminando & 3 & 33.3 \\
\hline & Carretilla & 2 & 22.2 \\
\hline & En burro y caminando & 1 & 11.1 \\
\hline & No contestó & 1 & 11.1 \\
\hline & Total & 9 & 100.0 \\
\hline
\end{tabular}

Fuente: Elaboración propia.

El número de viajes que las familias deben realizar por día para abastecerse de agua está en relación directa con los medios de transporte utilizados, ya que de ellos depende el volumen total que se puede obtener por viaje. Desde luego, con transporte animal se logra acarrear un volumen mucho mayor, llegando a cuadruplicar la cantidad de agua trasladada. Así, mientras las personas de Micuahutla, quienes no suelen usar los burros para el acarreo, deben realizar de cuatro a cinco viajes diarios a la cisterna colectiva para disponer del agua necesaria para sus diversas actividades domésticas y personales, en El Mirador, cuando hay un desperfecto de la bomba y se tiene que acarrear el agua desde el manantial, las familias van a la fuente una o, cuando mucho, dos veces al día.

Desde luego que el acarreo del agua es fundamental, dado que permite a las familias realizar sus actividades cotidianas, casi todas dependientes del vital líquido, y ello demanda tiempo y esfuerzo. Sin embargo, la pregunta aquí sería: ¿quiénes se responsabilizan de la realización de dicha actividad al interior de la familia? Ubicar a los actores sociales en este contexto es fundamental, al brindar información acerca del uso del tiempo y qué implicaciones tiene para el bienestar de las personas que lo hacen. Gran parte de la bibliografía al respecto asevera que la responsabilidad del acarreo recae principalmente en las mujeres (Melero, 2011; Molinares y Echeverría, 2011; Soares, 2007; Rico, 2006; Carmona, Alberti y Zapata, 1998) y los resultados encontrados en esta investigación no la contradicen, más bien abona a la tesis de que la división sexual del trabajo en zonas rurales sigue condicionando a las mujeres a la realización de las actividades relacionadas con el abasto del agua a las unidades domésticas, debido a que los hombres dedican mayor cantidad de tiempo a tareas remuneradas y las mujeres a las tareas domésticas, en las cuales el acceso al agua y al saneamiento es imprescindible; de tal suerte que la división del trabajo en el hogar, unida a los problemas en la prestación de servicios, refuerza las desigualdades de género.

EntreDiversidades. Revista de Ciencias Sociales y Humanidades, Vol. 8, Núm. 2 (17), julio-diciembre 2021. Páginas: 191-211 ISSN-e: 2007-7610. https://doi.org/10.31644/ED.V8.N2.2021.A09 
De hecho, el Cuadro 3 refleja lo enunciado, toda vez que las mujeres participan al 100\% en las actividades de acarreo, sean solas o en compañía de algún hombre de la familia (los hombres no acuden solos a la actividad de acarreo, sino siempre en compañía de mujeres).

\section{Cuadro 3. Quién realiza la actividad de acarreo del agua}

\begin{tabular}{|c|c|c|c|}
\hline Localidad & $\begin{array}{l}\text { Quién realiza la actividad de acarreo del } \\
\text { agua }\end{array}$ & Frecuencia & Porcentaje \\
\hline \multirow{7}{*}{ 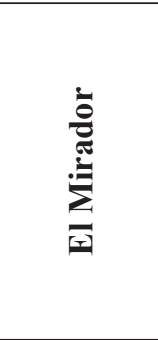 } & Mujeres adultas & 8 & 24.2 \\
\hline & Hombres y mujeres adultos & 9 & 27.3 \\
\hline & $\begin{array}{l}\text { Hombres y mujeres adultos y hombres } \\
\text { adolescentes }\end{array}$ & 2 & 6.1 \\
\hline & Todos(as) & 3 & 9.1 \\
\hline & Total & 22 & 66.7 \\
\hline & No opera & 11 & 33.3 \\
\hline & Total & 33 & 100.0 \\
\hline \multirow{6}{*}{ 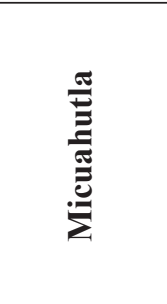 } & Mujeres adultas & 6 & 7 \\
\hline & Hombres y mujeres adultos & 1 & 11.1 \\
\hline & $\begin{array}{l}\text { Hombres y mujeres adultos y hombres } \\
\text { adolescentes }\end{array}$ & 1 & 11.1 \\
\hline & Total & 8 & 88.9 \\
\hline & No contestó & 1 & 11.1 \\
\hline & Total & 9 & 100.0 \\
\hline
\end{tabular}

Fuente: Elaboración propia.

A pesar que casi el 100\% de las viviendas de El Mirador cuenta con tubería para la distribución del agua - de hecho, solo una familia no accede al agua por el sistema-, también es cierto que el servicio no es continuo. Prueba de ello es que solamente 14 viviendas reportan recibir el agua los siete días de la semana y las demás 19 de uno a seis días, siendo que nueve familias la reciben tres días a la semana y a tres viviendas les tocan dos días. Además, la irregularidad del servicio de agua no se refiere solamente al número de días de la semana que el agua llega por la tubería, sino también a la cantidad de horas al día en que el agua está disponible en el sistema. De las 33 viviendas, 19 afirman recibir el agua las 24 horas del día, mientras que a las demás viviendas les llega de una a doce horas diarias. Ello se ve reflejado en la percepción del $47 \%$ de representantes de las viviendas, quienes afirman que tienen problemas para acceder al agua.

En El Mirador, la intermitencia en el servicio se debe a la carencia de recursos económicos para pagar la cuenta de luz a la Compañía Federal de Electricidad (CFE). La localidad gestiona el servicio a través de un Comité de Agua, cuyos miembros se encargan de recolectar entre todas las familias el recurso para pagar la cuenta de luz derivada del bombeo del agua. Además, esta irregularidad en el acceso al agua también está asociada a factores topográficos: a las viviendas más alejadas o ubicadas en los puntos más elevados de la localidad no les llega el mismo volumen de agua que a aquellas localizadas más cercanas a la cisterna de almacenamiento, causando en muchas ocasiones inconformidad y constituyéndose como punto potencial de conflictos por el agua. Además, las casas de la localidad cuentan con una serie de recipientes pequeńos para el 
almacenado de agua, debido a la carencia económica para la construcción de cisternas de elevada capacidad o la compra de tinacos.

Según Ilaya-Ayza, et al. (2015), entre los motivos por los cuales los sistemas de abastecimiento de agua no brindan el vital líquido de manera continua, están los siguientes: gestión técnica deficiente, carencia de recursos económicos o escasez física del recurso hídrico. Los autores añaden que los sistemas recurren a brindar el servicio de manera intermitente con el objetivo de reducir la demanda per cápita de agua y con ello lograr ahorros en costos de operación y de inversión; sin embargo, ello debilita la operación del sistema, con la reducción de sus niveles de presión, lo que trae como consecuencia la insuficiencia en el suministro en los puntos más alejados y/o elevados. Asimismo, dicha estrategia tiene un impacto directo en la economía doméstica de quienes padecen la intermitencia del servicio, dado que implica la compra de tinacos de almacenamiento de agua domiciliarios o, en su defecto, en las zonas de bajos ingresos, se recurre al almacenamiento en pequeños recipientes.

La problemática de la irregularidad en el servicio de agua no es ajena a la realidad de las localidades rurales de México, de hecho, constituye la regla y no la excepción, coincidente con otros países de la región latinoamericana, en donde se reporta que alrededor del $95 \%$ de los sistemas de abastecimiento rurales tienen problemas de intermitencia, mientras que el $60 \%$ del total de las viviendas de Latinoamérica y el Caribe, sean urbanas o rurales, poseen suministros de agua intermitentes. Asimismo, el Banco Mundial, en su informe Benchmarking Internacional de redes de agua y saneamiento, indica que el $84 \%$ de las empresas de agua en los países en vías de desarrollo no cumplen con la continuidad del servicio, brindando un promedio de servicio de 16 horas al día. Desde luego, esta desigualdad territorial en el acceso al agua, en donde las zonas rurales se encuentran en desventaja en comparación con las urbanas, constituye un obstáculo de primer orden no solo para el logro del ODS 6 de la Agenda 2030, sino para el pleno ejercicio de los derechos humanos (Nelson y Erickson, 2017; Ilaya-Ayza, et al., 2015; Vázquez-García, Pérez-Olvera y Muñoz-Rodríguez, 2014).

Asimismo, la totalidad de las viviendas de Micuahutla requiere de un esfuerzo constante para acceder al agua, de hecho, la mayoría realiza de cuatro a cinco viajes diarios a la cisterna colectiva. Estos datos coinciden con una investigación realizada por Ocampo y Villarreal (2014) en la región mixteca de Puebla, en donde la mayoría de las familias tiene que realizar de dos a tres viajes por día para lograr abastecerse de la demanda personal y doméstica de agua. Frente a la situación de dependencia de acarreo de agua, se suman otras condicionantes para su acceso. En estos términos, la disponibilidad de agua por persona en Micuahutla depende del número de habitantes en la vivienda, la edad y salud de sus integrantes y la utilización o no de animales como medio de transporte para el acarreo del agua.

Analizando los resultados encontrados en ambas localidades desde la perspectiva del ODS 6, encontramos severas deficiencias. Para la medición de la meta uno, relativa a la cobertura del agua potable, ${ }^{8}$ que es el tema que nos ocupa en esta contribución, el indicador se refiere a la "proporción de la población que dispone de servicios de suministro de agua potable gestionados

${ }^{8}$ La meta uno del ODS 6 está definida como: "De aquí a 2030, lograr el acceso universal y equitativo al agua potable a un precio asequible para todos" (ONU-Agua, 2017: 11).

EntreDiversidades. Revista de Ciencias Sociales y Humanidades, Vol. 8, Núm. 2 (17), julio-diciembre 2021. Páginas: 191-211 ISSN-e: 2007-7610. https://doi.org/10.31644/ED.V8.N2.2021.A09 
de manera segura" (ONU-Agua, 2017: 11). Dicho indicador está compuesto por tres variables: a) calidad (el agua no debe contener bacterias ni otro tipo de contaminantes), b) accesibilidad (el agua debe ubicarse in situ o en las inmediaciones) ${ }^{9}$, y c) disponibilidad (el agua debe estar disponible cuando se necesita). Vale mencionar que dichas variables corresponden con los criterios del derecho humano al agua, siendo que, además de estas, los derechos humanos contemplan la asequibilidad ${ }^{10}$ y la aceptabilidad, ${ }^{11}$ de tal suerte que, si bien los ODS se alinean con los derechos humanos, se quedan cortos en términos de indicadores. En relación con la variable uno, en ambas localidades no se cuenta con estudios sobre la calidad del agua, así que no hay datos al respecto, e incluso si se quisieran obtener la Conagua tampoco tendría la capacidad instalada para monitorear la calidad del agua de todas las comunidades rurales del país. Los municipios deberían hacerse cargo de la obtención de estos datos, sin embargo, no cuentan con los recursos humanos y financieros para ello, como es el caso de Zautla, cabecera municipal de las localidades de estudio. Mientras en El Mirador las personas confían en la calidad del agua de su manantial, justamente por su origen, en Micuahultla hay desconfianza y por ello suelen comprar garrafones.

En cuanto a la variable dos, relativa a la accesibilidad, en El Mirador se cumple si tomamos en cuenta que las viviendas disponen del servicio; sin embargo, cuando hay problemas con la bomba de agua, la comunidad suele quedar hasta tres meses seguidos sin el recurso hídrico en la tubería, por lo cual la población debe desplazarse hasta el manantial y el tiempo de acarreo es muy superior a los treinta minutos, por lo que en estas circunstancias se podría considerar que el servicio es "limitado". ${ }^{12}$ En Micuahutla, con viviendas dispersas, el acarreo desde la cisterna colectiva puede llegar a ser superior a los treinta minutos para algunas familias, por lo cual la localidad en su totalidad no necesariamente cumpliría con esta condición y, al igual que El Mirador, podría clasificarse como de servicio "limitado".

Finalmente, en lo concerniente a la disponibilidad, mientras en El Mirador, que cuenta con el servicio de agua, el suministro es intermitente, en Micuahutla el abasto se hace exclusivamente a través del desplazamiento de integrantes de la unidad doméstica a la cisterna colectiva, por lo que el volumen que se logra obtener por vivienda depende enteramente de los viajes a la cisterna. Por tanto, en ambas localidades no se cumple la variable de "disponibilidad", cuya definición desde el derecho humano al agua establece que se requiere de abastecimiento continuo

\footnotetext{
${ }^{9} \mathrm{El}$ agua potable de una fuente mejorada que no está dentro de la vivienda se clasifica como servicio "básico" si el trayecto de ida y vuelta para recoger el agua no supera los 30 minutos, incluida la correspondiente fila de espera. Si la fuente de agua potable mejorada se sitúa más lejos, se considera que el servicio es “limitado” (ONU-Agua, 2017: 11).

${ }^{10} \mathrm{La}$ asequibilidad, también conocida como accesibilidad económica, se refiere a que los costos asociados con el abastecimiento de agua no deben comprometer el ejercicio de otros derechos. La OMS establece como valor de referencia, para que el acceso al agua y al saneamiento no sea un obstáculo para las familias más pobres, que el costo de dichos servicios no sea superior al 3\% del ingreso total familiar (Albuquerque, 2014).

${ }^{11} \mathrm{La}$ aceptabilidad está estrechamente relacionada con las normas socioculturales que rigen los grupos sociales, de tal suerte que las instalaciones y servicios de agua deben ser aceptables desde el punto de vista cultural (Albuquerque, 2014).

${ }^{12} \mathrm{El}$ servicio limitado se ubica en un rango bastante inferior al "deseable", toda vez que el indicador puede desglosarse por nivel de servicio: sin servicios, servicios limitados, servicios básicos y finalmente lo ideal, servicios gestionados de manera segura (ONU-Agua, 2017).
}

EntreDiversidades. Revista de Ciencias Sociales y Humanidades, Vol. 8, Núm. 2 (17), julio-diciembre 2021. Páginas: 191-211 ISSN-e: 2007-7610. https://doi.org/10.31644/ED.V8.N2.2021.A09 
y suficiente para los usos personales y domésticos, que incluyen ingesta directa, preparación de alimentos, saneamiento personal, lavado de ropa, así como higiene personal y doméstica (ONU, 2010). Así, podemos concluir, con los datos presentados, que ambas comunidades no cumplen con el ODS 6 en su meta uno ni con el ejercicio del derecho humano al agua. Aunque exista un esfuerzo sustancial del gobierno mexicano para promover el ejercicio progresivo del derecho humano al agua, y con ello el cumplimiento del ODS 6, en su meta uno, en todo caso los resultados se plasmarían a mediano o largo plazo, en el supuesto de poder lograrse y en virtud de las severas limitaciones estructurales en la gestión del agua.

\section{Conclusiones}

Esta investigación, realizada con fines comparativos en dos comunidades rurales del estado de Puebla, analiza el acceso, disponibilidad y calidad del agua para las familias, a fin de brindar elementos sobre los desafíos que implican el cumplimiento de la Agenda 2030 en México, en lo relativo al agua, en especial en comunidades rurales. El lema de la Agenda 2030 de Desarrollo Sostenible es "no dejar a nadie atrás", sin embargo, las personas que habitan los territorios rurales ya quedaron atrás y remontar esta desigualdad no implica un esfuerzo menor. Los objetivos de la agenda mencionada serían alcanzables en la medida en que exista una voluntad política y recursos económicos que derive en renovados esfuerzos para incluir a aquellas personas y localidades "que se quedaron atrás" en los procesos de desarrollo.

En el desarrollo de la investigación, con recolección de información acerca de las variables de acceso, disponibilidad y calidad del agua, relativos al ODS 6 en su meta uno, se puso en evidencia las actividades o medidas que deben ser tomadas por las localidades estudiadas para suplir las necesidades de agua de las viviendas. Para lograr entender mejor el acceso y disponibilidad del recurso hídrico por las familias se indagó sobre los medios de transporte utilizados para el abasto del agua y quiénes realizan la actividad de acarreo.

Mientras El Mirador combina la red domiciliaria, acarreo desde el manantial y cosecha de agua de lluvia como fuentes de abastecimiento de agua, en Micuahutla las viviendas se abastecen vía una cisterna colectiva, captura de agua de lluvia y compra de garrafones. En cuanto a los medios de transporte del agua a la vivienda, las familias de El Mirador utilizan predominantemente la tracción animal, y las de Micuahutla la obtienen acudiendo a la cisterna colectiva a pie. Cabe aclarar que, mientras en El Mirador el acarreo es una actividad esporádica cuando hay desperfecto en la bomba que suministra el agua al sistema de tuberías, en Micuahutla es una actividad permanente, dado que no cuenta con un sistema formal de agua. En ambas localidades son las mujeres quienes principalmente se encargan del abasto de agua, como responsables de todas las tareas domésticas.

Esto pone en situación de vulnerabilidad a la población que vive en dichas localidades, al no contar con una garantía de acceso al servicio que brinde un suministro suficiente y regular de agua. De hecho, los resultados evidencian una constante inseguridad en el acceso al agua, principalmente en la localidad de Micuahutla, que carece de una fuente de agua y depende de la voluntad del ayuntamiento municipal para el envío de camiones pipa, lo que limita o imposibilita el desarrollo de actividades agropecuarias, por lo cual la población de la localidad se encuentra

EntreDiversidades. Revista de Ciencias Sociales y Humanidades, Vol. 8, Núm. 2 (17), julio-diciembre 2021. Páginas: 191-211 ISSN-e: 2007-7610. https://doi.org/10.31644/ED.V8.N2.2021.A09 
en situación de vulnerabilidad extrema, perpetuando sus condiciones de marginación social. La calidad el agua es una incógnita en ambas localidades, pues no se cuenta con datos al respecto, sin embargo, mientras en El Mirador la población tiene confianza en la buena calidad de su agua, al argumentar que es de "manantial", en Micuahutla prefieren comprar garrafones para no arriesgar su salud, dado que no confían en la calidad del agua para consumo personal.

Con el análisis de los resultados de esta investigación podemos concluir que ambas comunidades no cumplen con el ODS 6 en su meta uno ni con el ejercicio del derecho humano al agua. Ello tiene implicaciones directas sobre las oportunidades de las personas, dado que deben hacer un esfuerzo y utilizar su tiempo disponible para la obtención del agua, reduciendo sus posibilidades de desarrollo. Asimismo, hay que mencionar el impacto negativo del limitado acceso y disponibilidad del agua en la salud y en la retroalimentación del ciclo vicioso de la pobreza y la desigualdad. En virtud de esta situación es imprescindible diseñar nuevas estrategias para la gestión y el manejo de aguas en México, reconociendo al derecho humano al agua como la guía orientadora de la gestión. Ya contamos con un avance importante en la materia, relativo al reconocimiento explícito del derecho humano al agua en nuestra Constitución, sin embargo, aún falta un camino por recorrer, relativo a la elaboración de leyes y reglamentos para su cumplimiento y una efectiva participación ciudadana para acompańar y exigir su observancia. Reconocer el derecho humano al agua llevaría a una distribución más equilibrada del recurso hídrico y contribuiría en la reducción de las desigualdades, beneficiando a poblaciones que tradicionalmente han sido marginadas.

La reducción de las brechas de desigualdad en términos de cobertura del servicio de agua entre zonas rurales y urbanas debe ser prioritaria, debido a la necesidad de atender a los grupos más vulnerables. De hecho, así lo plantea la Agenda 2030 para el Desarrollo Sostenible. Ello invita a una reflexión sobre la relación entre desigualdades, derechos humanos y ODS, en específico en lo referente al agua. Prevalece en México una realidad preocupante en términos de desigualdad en el acceso al agua, en donde las zonas rurales son las más perjudicadas, y vale recordar los vínculos entre el limitado acceso al agua y la pobreza, su impacto sobre la salud, la producción de alimentos y la igualdad de género, conllevando en definitiva a la exclusión social. Por ello, el ejercicio del derecho humano al agua contribuiría no solamente al cumplimiento del objetivo 6 de los ODS, sino al logro de un amplio conjunto de objetivos, entre ellos el fin de la pobreza (ODS 1), hambre cero (ODS 2), salud y bienestar (ODS 3), igualdad de género (ODS 5) y reducción de las desigualdades (ODS 10). En definitiva, mientras perdure la desigualdad territorial y marginación social, no se logrará garantizar el cumplimiento del ODS 6 ni el ejercicio del derecho humano al agua, por lo que las desigualdades siguen constituyendo un factor determinante de la vulnerabilidad e incertidumbre que enfrentan los grupos sociales en su articulación con el agua.

Agradecimientos: se agradece al Fondo CONACYT - FORDECYT de México, por brindar la oportunidad del desarrollo del proyecto: "Modelo interdisciplinario para ejercer el derecho humano al agua y al saneamiento en zonas rurales marginadas de México”.

EntreDiversidades. Revista de Ciencias Sociales y Humanidades, Vol. 8, Núm. 2 (17), julio-diciembre 2021. Páginas: 191-211 ISSN-e: 2007-7610. https://doi.org/10.31644/ED.V8.N2.2021.A09 


\section{Bibliografía citada}

Albuquerque, Catarina de (2014). Manual práctico para la realización de los derechos humanos al agua y al saneamiento. Lisboa, Portugal: Agencia Española de Cooperación Internacional para el Desarrollo, Auswärtiges Amt, Swiss Agency for Development and Cooperation, Ministry for Foreign Affairs for Finland, The Water and Waste Services Regulation Authority, WaterAid, Fondo de las Naciones Unidas para la Infancia, Programa de Naciones Unidas para los Asentamientos Humanos. Disponible en: https://www.ohchr. org/Documents/Issues/Water/Handbook/Book1 intro sp.pdf (Consultado el 15 de abril de 2021).

Carmona, Edith, Pilar Alberti y Emma Zapata (1998). "Acceso y uso del agua por las campesinas en la unidad doméstica y la parcela agrícola de riego. Alto Río Lerma, Guanajuato". Comunicaciones en Socioeconomía, Estadistica e Informática, 2 (6), pp. 5-25.

Consejo Nacional de Evaluación de la Política de Desarrollo Social (CONEVAL) (2020). Preguntas derivadas del curso-taller metodología oficial para la medición multidimensional de la pobreza [en línea]. Disponible en: https://www.coneval.org.mx/Eventos/Paginas/ preguntas-curso.aspx (Consultado el 16 de abril de 2020).

Fernández L., Juan, María Ignacia Fernández e Isidro Soloaga (2019). Enfoque territorial y análisis dinámico de la ruralidad: alcances y límites para el diseño de políticas de desarrollo rural innovadoras en América Latina y el Caribe. Documentos de Proyectos. Ciudad de México, México: Comisión Económica para América Latina y el Caribe. Disponible en: https:// repositorio.cepal.org/bitstream/handle/11362/44905/1/S1900977 es.pdf (Consultado el 22 de agosto de 2020).

García Dávila, Alejandrina y Vázquez García, Verónica (2017). "Derecho humano al agua y desigualdad social en San Jerónimo Tecóatl, Oaxaca”. Cuicuilco Revista de Ciencias Antropológicas [en línea], 24 (68), pp. 157-176. Disponible en: https://www.revistas.inah. gob.mx/index.php/cuicuilco/article/view/11282 (Consultado el 27 de agosto de 2020).

Gobierno de la República (2018). Informe Nacional Voluntario para el Foro Politico de Alto Nivel sobre Desarrollo Sostenible. Bases y fundamentos en México para una visión del desarrollo sostenible a largo plazo. Avance en el cumplimiento de la Agenda 2030 y los Objetivos de Desarrollo Sostenible [en línea]. Disponible en: https://sustainabledevelopment.un.org/ content/documents/20125INFORME NACIONAL VOLUNTARIO 060718.pdf (Consultado el 15 de abril de 2020).

Gutiérrez Villalpando, Verónica, et al. (2013). "Mujeres y organización social en la gestión del agua para consumo humano y uso doméstico en Berriozábal, Chiapas”. LiminaR. Estudios Sociales y Humanisticos [en línea], 11 (2), pp. 100-113. doi: https://doi.org/10.29043/ liminar.v11i2.225 (Consultado el 21 de mayo de 2021).

Hortelano Villanueva, Lucía e Hidalgo García, María del Mar (2016). El agua como derecho humano. Retos y limitaciones. Documento de Análisis [en línea]. Madrid, España: Instituto Español de Estudios Estratégicos. Disponible en: http://www.ieee.es/Galerias/fichero/ docs analisis/2016/DIEEEA78-2016 DerechoAgua MMHG.pdf (Consultado el 29 de julio de 2020).

EntreDiversidades. Revista de Ciencias Sociales y Humanidades, Vol. 8, Núm. 2 (17), julio-diciembre 2021. Páginas: 191-211 ISSN-e: 2007-7610. https://doi.org/10.31644/ED.V8.N2.2021.A09 
Ilaya-Ayza, Amilkar Ernesto, et al. (2015). "La problemática de los sistemas de suministro de agua intermitentes. Aspectos generales”. RIOC Revista Ingeniería de Obras Civiles [en línea], Vol. 5, pp. 31-39. Disponible en: http://revistas.ufro.cl/ojs/index.php/rioc/article/ view/1990 (Consultado el 29 de julio de 2020).

Instituto Nacional de Estadística y Geografía (INEGI) (2016). Encuesta Intercensal 2015 [en línea]. Disponible en: https://www.inegi.org.mx/programas/intercensal/2015/ (Consultado el 10 de agosto de 2020).

Instituto Nacional para el Federalismo y el Desarrollo Municipal (INAFED) (2020). Enciclopedia de los municipios y delegaciones de México. Estado de Puebla. Zautla [en línea]. Disponible en: http://www.inafed.gob.mx/work/enciclopedia/EMM21puebla/municipios/21212a. html (Consultado el 18 de abril de 2020).

Jusidman, Clara (2009). "Desigualdad y política social en México". Nueva Sociedad [en línea], Núm. 220, pp. 190-206. Disponible en: https://nuso.org/articulo/desigualdad-ypolitica-social-en-mexico/ (Consultado el 28 de abril de 2020).

Llanos-Hernández, Luis (2010). "El concepto del territorio y la investigación en las ciencias sociales". Agricultura, sociedad y desarrollo [en línea], 7 (3), septiembre-diciembre, pp. 207-220. Disponible en: http://www.scielo.org.mx/pdf/asd/v7n3/v7n3a1.pdf (Consultado el 19 de mayo de 2021).

López Morales, Carlos A. (2017). "El estado del agua en México: retos, oportunidades y perspectivas", en Denzin, Christian, Federico Taboada y Raúl Pacheco-Vega (eds.). El agua en México. Actores, sectores y paradigmas para una transformación social-ecológica [en línea]. Ciudad de México, México: Fundación Friedrich Ebert, pp. 13-42. Disponible en: https://agua.org.mx/biblioteca/el-agua-en-mexico-actores-sectores-y-paradigmaspara-una-transformacion-social-ecological (Consultado el 27 de agosto de 2020).

Melero Aguilar, Noelia (2011). "La participación de la mujer en el acceso y gestión del agua. Una experiencia cubana”. Anduli. Revista Andaluza de Ciencias Sociales [en línea], Núm. 10, pp. 21-30. Disponible en: https://revistascientificas.us.es/index.php/anduli/issue/ view/369 (Consultado el 25 de agosto de 2020).

Molinares Hassan, Viridiana y Echeverría Molina, Judith (2011). "El derecho humano al agua: posibilidades desde una perspectiva de género". International Law: Revista Colombiana de Derecho Internacional [en línea], 9 (19), pp. 269-302. Disponible en: https://revistas. javeriana.edu.co/index.php/internationallaw/article/view/13740 (Consultado el 25 de agosto de 2020).

Nelson, Kara L. y Erickson, John (2017). Suministro intermitente en el contexto de esfuerzos por mejorar el abastecimiento de agua potable en América Latina y el Caribe. Lecciones de un estudio de caso en Arraiján, Panamá. Banco Interamericano de Desarrollo [en línea]. Disponible en: https://publications.iadb.org/publications/spanish/document/Suministro-intermitenteen-el-contexto-de-esfuerzos-por-mejorar-el-abastecimiento-de-agua-potableen-Am\%C3\%A9rica-Latina-y-el-Caribe-Lecciones-de-un-estudio-de-caso-enArraij\%C3\%A1n-Panam\%C3\%A1.pdf (Consultado el 7 de abril de 2020).

EntreDiversidades. Revista de Ciencias Sociales y Humanidades, Vol. 8, Núm. 2 (17), julio-diciembre 2021. Páginas: 191-211 ISSN-e: 2007-7610. https://doi.org/10.31644/ED.V8.N2.2021.A09 
Nogueira, Daniela, Carolina Milhorance y Priscylla Mendes (2020). “Do Programa Um Milhão de Cisternas ao Água para Todos: divergências políticas e bricolagem institucional na promoção do acesso à água no Semiárido brasileiro". IdeAs [en línea], Núm. 15. doi: https://doi.org/10.4000/ideas.7219 (Consultado el 18 de abril de 2020).

Ocampo Fletes, Ignacio y Villarreal Manzo, Luis A. (2014). "Recursos hídricos, movilidad social territorial para su aprovechamiento y derecho humano al agua en comunidades de la mixteca baja de Puebla, México". Ambiente y Desarrollo [en línea], 18 (35), pp. 55-69. doi: https://doi.org/10.11144/Javeriana.AyD18-35.rhms (Consultado el 21 de agosto de 2020).

Oficina del Alto Comisionado de las Naciones Unidas para los Derechos Humanos (OHCHR) (2017). Declaración final de misión del Relator Especial sobre los derechos humanos al agua y al saneamiento, Sr. Léo Heller [en línea]. Disponible en: https://www.ohchr.org/SP/ NewsEvents/Pages/DisplayNews.aspx?NewsID=21608\&LangID=S (Consultado el 14 de abril de 2020).

Oliveira, Alynne de, et al. (2020). "Una mirada crítica a los objetivos de desarrollo sostenible a partir de una experiencia realizada por estudiantes de primaria: ¿ser o no ser, esa es la cuestión?". Pensamiento educativo. Revista de Investigación Educacional Latinoamericana [en línea], 57 (2), pp. 1-23. doi: https://doi.org/10.7764/PEL.57.2.2020.7 (Consultado el 21 de mayo de 2021).

Organización Mundial de la Salud (OMS) y Fondo de las Naciones Unidas para la Infancia (UNICEF) (2017). Progresos en materia de agua potable, saneamiento e higiene: informe de actualización de 2017 y línea de base de los ODS [en línea]. Ginebra, Suiza: Organización Mundial de la Salud y Fondo de las Naciones Unidas para la Infancia (UNICEF). Disponible en: https://apps.who.int/iris/handle/10665/260291 (Consultado el 17 de agosto de 2020).

Organización de Naciones Unidas (ONU) (2010). El derecho bumano al agua y al saneamiento [en línea]. Disponible en: https://www.un.org/spanish/waterforlifedecade/human right to water.shtml (Consultado el 20 de abril de 2020).

ONU-Agua (2017). Guía para el monitoreo integrado del Objetivo de Desarrollo Sostenible Seis. Metas e indicadores mundiales [en línea]. Disponible en: https://www.unwater.org/app/ uploads/2017/10/G2 Metas-e-indicadores-mundiales Version-2017-07-14.pdf (Consultado el 22 de abril de 2020).

Peña, Francisco y Pérez, Ricardo (2016). "Abasto de agua y geografía de la desigualdad urbana. Periferia social y bienes públicos impuros", en Santacruz de León, Germán y Peña, Francisco (coords.). Problemática y desigualdad en la gestión del agua de la cuenca semiárida y urbanizada del Valle de San Luis Potosí. San Luis Potosí, México: El Colegio de San Luis, pp. 19-26.

EntreDiversidades. Revista de Ciencias Sociales y Humanidades, Vol. 8, Núm. 2 (17), julio-diciembre 2021. Páginas: 191-211 ISSN-e: 2007-7610. https://doi.org/10.31644/ED.V8.N2.2021.A09 
Programa Mundial de Evaluación de los Recursos Hídricos de la UNESCO (WWAP) (2019). Informe Mundial de las Naciones Unidas sobre el Desarrollo de los Recursos Hidricos 2019: No dejar a nadie atrás [en línea]. París, Francia: Organización de las Naciones Unidas para la Educación, la Ciencia y la Cultura. Disponible en: https://es.unesco.org/watersecurity/wwap/wwdr/2019 (Consultado el 11 de agosto de 2020).

Rico, María Nieves (2006). "Género y agua”, en Esch, Sofía, et al. (eds.). La gota de la vida. Hacia una gestión sustentable y democrática del agua. México, D.F., México: Ediciones Böll, pp. 255-264.

Sandoval Minero, Ricardo (2017). "El agua en la agenda 2030 y su relación con los Objetivos del Desarrollo Sostenible", en Denzin, Christian, Federico Taboada y Raúl PachecoVega (eds.). El agua en México. Actores, sectores y paradigmas para una transformación social-ecológica. Ciudad de México, México: Fundación Friedrich Ebert, pp. 123-147. Disponible en: https://agua.org.mx/biblioteca/el-agua-en-mexico-actores-sectores-yparadigmas-para-una-transformacion-social-ecologica/ (Consultado el 10 de agosto de 2020).

Sandoval Terán, Areli y Seguin Tovar, Nathalie (2018). Los ODS en México sin buenas perspectivas al final del sexenio [en línea]. Disponible en: https://www.socialwatch.org/node/18094 (Consultado el 20 de abril de 2020).

SecretaríadeDesarrolloSocial(SEDESOL) (2013). UnidaddeMicrorregiones. CédulasdeInformación Municipal. Municipio Zautla [en línea]. Disponible en: http://www.microrregiones.gob. $\underline{\mathrm{mx} / \text { catloc/LocdeMun.aspx?tipo=clave } \& \mathrm{campo}=\operatorname{loc} \& e n t=21 \& \mathrm{mun}=212}$ (Consultado el 16 de abril de 2020).

Secretaría de Medio Ambiente y Recursos Naturales (SEMARNAT) y Comisión Nacional del Agua (CONAGUA) (2018). Estadísticas del Agua en México 2018 [en línea]. Ciudad de México, México: Secretaría de Medio Ambiente y Recursos Naturales y Comisión Nacional del Agua. Disponible en: http://sina.conagua.gob.mx/publicaciones/EAM 2018.pdf (Consultado el 7 de abril de 2020).

Soares, Denise (2007). "Acceso, abasto y control del agua en una comunidad indígena chamula en Chiapas. Un análisis a través de la perspectiva de género, ambiente y desarrollo”. región y sociedad [en línea], 19 (38), pp. 25-50. doi: https://doi.org/10.22198/rys.2007.38.a556 (Consultado el 11 de abril de 2021).

Varela, Micaela, Héctor Guerrero y Teresa de Miguel (2021). "La sequía que abrasa México. Una tragedia predecible y devastadora”. El Pais [en línea]. Disponible en: https://elpais. com/mexico/2021-04-24/la-sequia-que-abrasa-mexico-una-tragedia-predecible-ydevastadora.html (Consultado el 18 de mayo de 2021).

EntreDiversidades. Revista de Ciencias Sociales y Humanidades, Vol. 8, Núm. 2 (17), julio-diciembre 2021. Páginas: 191-211 ISSN-e: 2007-7610. https://doi.org/10.31644/ED.V8.N2.2021.A09 
Vargas, Sergio, Alejandra Peña y Denise Soares (2018). "Urbanización y gestión del agua ante el cambio climático, en el área metropolitana de Cuernavaca”, en Soares, Denise y Peńa, Alejandra (coords.). Impacto del cambio climático para la gestión integral de la cuenca hidrológica del río Apatlaco. Jiutepec, Morelos, México: Secretaría del Medio Ambiente y Recursos Naturales, Instituto Mexicano de Tecnología del Agua, pp. 377-421. Disponible en: http://www.imta.gob.mx/biblioteca/libros html/rio apatlaco/cambio climatico rio aplatlaco.pdf (Consultado el 10 de abril de 2021).

Vázquez-García, Verónica, María Antonia Pérez-Olvera y Carolina Muñoz-Rodríguez (2014). "Desarrollo, género y el derecho humano al agua. Un estudio comparativo en Hidalgo, México". Agricultura, Sociedad y Desarrollo [en línea], 11 (3), pp. 295-314. doi: https:// doi.org/10.22231/asyd.v11i3.82 (Consultado el 7 de abril de 2021). 